\title{
Need for relativistic corrections in the analysis of spatial adiabatic passage of matter waves
}

\author{
A. Benseny, ${ }^{1}$ J. Bagudà, ${ }^{1}$ X. Oriols, ${ }^{2}$ and J. Mompart ${ }^{1}$ \\ ${ }^{1}$ Departament de Física, Universitat Autònoma de Barcelona, E-08193 Bellaterra, Spain \\ ${ }^{2}$ Departament d' Enginyeria Electrònica, Universitat Autònoma de Barcelona, E-08193 Bellaterra, Spain
}

(Received 21 July 2011; published 15 May 2012)

\begin{abstract}
We investigate the coherent transport of a single particle and a Bose-Einstein condensate between the two extreme traps of a triple-well potential by means of the spatial adiabatic passage technique. This matter wave transport technique consists of adiabatically following an energy eigenstate of the system that only populates the vibrational ground states of the two extreme wells and presents at all times a node in the central region. Unraveling the (nonlinear) time-dependent Schrödinger equation in terms of Bohmian quantum trajectories, we show that by slowing down the total time duration of the transport process, Bohmian velocities in the central region are orders of magnitude larger than the mean atomic velocities. This leads to a very counterintuitive effect: in the regime of almost perfect adiabaticity, these velocities require relativistic corrections to properly address the transfer process and avoid superluminal propagation.
\end{abstract}

DOI: 10.1103/PhysRevA.85.053619

PACS number(s): 03.75.-b, 05.60.Gg, 67.85.-d

\section{INTRODUCTION}

Classical laws govern all of our daily physical situations providing us with an intuition on what is physically feasible. It is not surprising then that a wide collection of quantum and relativistic phenomena escape from our "limited" classical intuition such as the well-known Schrödinger's cat and Einstein-Podolsky-Rosen quantum paradoxes, or the tunnel and twin paradoxes in the relativistic realm. Their detailed analysis has allowed physicists to achieve a deeper understanding of quantum mechanics and relativity. Within this context, we will present and discuss in this article a very counterintuitive behavior that appears in the adiabatic transport of ultracold atoms between the two extreme wells of a triple-well potential: in the regime of almost perfect adiabaticity, relativistic corrections are needed to properly address the transport dynamics even though the associated average velocities of the system are much smaller than the speed of light.

Stimulated Raman adiabatic passage (STIRAP) [1] is a quantum-optical technique used to transfer electronic population between two internal levels of an atom or a molecule where these two levels are coupled via two delayed laser pulses through an intermediate level that is (ideally) never populated during the process. The matter wave analog of STIRAP via the tunneling interaction, the spatial adiabatic passage (SAP) technique, has been proposed to transfer either single particles [2-5] or Bose-Einstein condensates (BECs) [6,7] between the two extreme traps of a triple-well potential. The SAP technique consists of transporting a matter wave by adiabatically following an energy eigenstate of the system, the spatial dark state, that only populates the vibrational ground states of the two extreme wells and presents at all times a node in the central region.

The adiabatic following of a spatial dark state, albeit being analogous to the STIRAP technique, presents significant differences that have unexpected consequences: while STIRAP refers to the transfer of population between two internal levels, the SAP technique implies matter wave transport between two distant regions separated by a quasinode. In fact, while the matter wave is transferred between the outer traps, the population in the central one can be made arbitrarily small at any time of the evolution by slowing down the process [7]. It has been recently shown analytically that the small central well population during the SAP process is due to the high current (or velocity) in this region [3]. Just as a (classical) fluid increases its velocity when passing through a narrow conduct, as the SAP is performed more slowly and the probability density at the central well decreases, its associated velocity increases. If so, one could naively (and erroneously) conclude that an instantaneous atomic transport from the left to the right trap can appear in the limit of perfect adiabaticity. However, the quantum continuity equation forbids matter teleportation, and, as we will show, relativistic corrections become necessary to describe the system evolution for very large transport times.

In this article we numerically investigate the adiabatic transport of a single atom and a BEC in a triple-well potential by unraveling the (nonlinear) time-dependent Schrödinger equation (TDSE) in terms of Bohmian trajectories [8,9]. Bohmian mechanics is equivalent to standard quantum mechanics, given that the trajectories are randomly distributed following the modulus squared of the wave function, and provides a very clear physical picture of the quantum dynamics. Since, with a correct relativistic treatment, Bohmian trajectories for massive particles cannot surpass the speed of light [9-11], the appearance of superluminal Bohmian velocities is an irrefutable indication of an incorrect calculation of Bohmian trajectories, and therefore the consequence of the application of the TDSE in a regime where it is not valid. Thus, our main motivation in using Bohmian trajectories to investigate the problem at hand is that they are very well indicated to elucidate whether relativistic corrections are needed to properly describe the dynamics of a particular quantum system. With this in mind, we will show that as the SAP process is performed more adiabatically, the velocity in the central region increases with no apparent limit such that, in the regime of almost perfect adiabaticity, the nonrelativistic TDSE does not correctly describe the spatial adiabatic transport of cold atoms.

The appearance of superluminal Bohmian velocities near a wave-function node is not an exclusive characteristic of the dynamical system discussed in this paper. Any system with 
nodes might exhibit similar features, but this does not imply, in general, any problem since such superluminal Bohmian trajectories are marginal because the nodes are not relevant to the dynamics. In the system we discuss here, however, the (quasi)node plays a crucial role in the dynamics since all Bohmian trajectories are forced to transit through it.

\section{PHYSICAL SYSTEM}

The nonlinear TDSE or Gross-Pitaevskii equation (GPE) that describes the dynamics of a BEC reads

$i \hbar \frac{\partial \psi(\vec{r}, t)}{\partial t}=\left[-\frac{\hbar^{2}}{2 m} \nabla^{2}+V(\vec{r}, t)+g|\psi(\vec{r}, t)|^{2}\right] \psi(\vec{r}, t)$,

where $\psi$ is the wave function of the BEC, $m$ is the mass of the BEC atoms, $V$ is the trapping potential, and $g$ governs the nonlinear interaction between the atoms [12]. For $g=0$ we recover the standard TDSE that deals with the dynamics of a single atom or a noninteracting BEC.

In Bohmian mechanics [8,9], $\psi(\vec{r}, t)$ guides an infinite ensemble of trajectories described by a flow map, $\overrightarrow{\mathrm{r}}\left[t, \vec{r}_{0}\right]$, which maps each possible initial position $\vec{r}_{0}$ (at $t=0$ ) to a later position $\overrightarrow{\mathrm{r}}\left[t, \vec{r}_{0}\right]$ at time $t$ along its trajectory

$$
\begin{gathered}
\overrightarrow{\mathrm{r}}\left[t, \vec{r}_{0}\right]=\vec{r}_{0}+\left.\int_{0}^{t} d t^{\prime} \vec{v}\left(\vec{r}, t^{\prime}\right)\right|_{\vec{r}=\overrightarrow{\mathrm{r}}\left[t^{\prime}, \vec{r}_{0}\right]}, \\
\vec{v}(\vec{r}, t) \equiv \frac{\vec{J}(\vec{r}, t)}{|\psi(\vec{r}, t)|^{2}}=\frac{1}{m} \vec{\nabla} S(\vec{r}, t) .
\end{gathered}
$$

$\vec{J}$ is the probability current density and $S$ is the wavefunction phase. To simplify our notation, we define $\overrightarrow{\mathrm{v}}\left[t, \vec{r}_{0}\right] \equiv$ $d \overrightarrow{\mathrm{r}}\left[t, \vec{r}_{0}\right] / d t$ as the velocity of the Bohmian trajectories. As long as the initial trajectory distribution follows $\left|\psi\left(\vec{r}_{0}, 0\right)\right|^{2}$, the trajectory ensemble reproduces the probability and current densities at any time

$$
\begin{gathered}
|\psi(\vec{r}, t)|^{2}=\int_{-\infty}^{\infty} d \vec{r}_{0}\left|\psi\left(\vec{r}_{0}, 0\right)\right|^{2} \delta\left(\vec{r}-\overrightarrow{\mathrm{r}}\left[t, \vec{r}_{0}\right]\right), \\
\vec{J}(\vec{r}, t)=\int_{-\infty}^{\infty} d \vec{r}_{0}\left|\psi\left(\vec{r}_{0}, 0\right)\right|^{2} \overrightarrow{\mathrm{v}}\left[t, \vec{r}_{0}\right] \delta\left(\vec{r}-\overrightarrow{\mathrm{r}}\left[t, \vec{r}_{0}\right]\right),
\end{gathered}
$$

and thus, averages over the trajectory ensemble are equivalent to mean values obtained from the wave function. Furthermore, by inserting the wave function in polar form (i.e., $\psi=$ $\left.|\psi| e^{i S / \hbar}\right)$ in the GPE and separating real and imaginary parts we obtain

$$
\begin{aligned}
& \frac{\partial S}{\partial t}=-\left(V+g|\psi|^{2}+\frac{1}{2} m \vec{v}^{2}-\frac{\hbar^{2}}{2 m} \frac{\nabla^{2}|\psi|}{|\psi|}\right), \\
& \frac{\partial|\psi|^{2}}{\partial t}=-\vec{\nabla} \cdot\left(|\psi|^{2} \vec{v}\right) .
\end{aligned}
$$

Equation (6) is the quantum Hamilton-Jacobi equation, analogous to its classical counterpart, but with an additional term, the quantum potential [9], which accounts for the quantum features of the system. Equation (7) is the quantum continuity equation for the condensate density that ensures that transport is always carried out in a continuous fashion and that Eqs. (4) and (5) are verified at all times [9] [i.e., that the ensemble of trajectories reproduces exactly the wave function given by Eq. (1)]. Therefore, if the system description given by either the (nonrelativistic) Bohmian trajectories or the TDSE is incorrect (for instance, the appearance of superluminal
Bohmian velocities [10]), the description due to the other one must also be incorrect.

To ease the computational burden we will work with the reduction of Eqs. (1) through (7) to one dimension, but the entire discussion here is also valid for the full threedimensional case. Following Ref. [7], we consider a triple-well potential consisting of a harmonic trap of frequency $\omega_{x}$ (and ground-state width $\alpha^{-1} \equiv \sqrt{\hbar / m \omega_{x}}$ ) where two Gaussian barriers of heights $V_{12}$ and $V_{23}$ have been added at positions $\mp x_{b}$, giving a total potential of the form

$$
V(x, t)=\frac{m}{2} \omega_{x}^{2} x^{2}+V_{12}(t) e^{-\frac{\left(x+x_{b}\right)^{2}}{2 \sigma^{2}}}+V_{23}(t) e^{-\frac{\left(x-x_{b}\right)^{2}}{2 \sigma^{2}}} .
$$

The barriers, created, for instance, with two blue-detuned lasers, will be considered initially high enough to inhibit tunneling such that three distinct regions are well defined: $\mathcal{C}_{1}=$ $\left(-\infty,-x_{b}\right), \mathcal{C}_{2}=\left(-x_{b}, x_{b}\right)$, and $\mathcal{C}_{3}=\left(x_{b}, \infty\right)$. The eventual lowering of the barriers allows the BEC, located initially in the left trap, to tunnel to the other trapping regions. The population in each region can be computed as $P_{i}(t)=\int_{\mathcal{C}_{i}} d x|\psi(x, t)|^{2}$. For the initial state (i.e., the numerically computed ground state of the left well), we have $P_{2}(0)+P_{3}(0)<10^{-14}$, and therefore all (relevant) trajectories will start in region $\mathcal{C}_{1}$ (i.e., the contribution of trajectories starting in regions 2 and 3 to averages is negligible).

The adiabatic transport of the BEC from the left to the right trap is achieved by following an energy eigenstate of the system, the spatial dark state, which only involves the ground states of the left and right traps, namely $|L\rangle$ and $|R\rangle$, and presents a node in the middle region. The dark state reads $|D(\theta)\rangle=\cos \theta|L\rangle-\sin \theta|R\rangle$, where the mixing angle $\theta$ is $\tan \theta \equiv \Omega_{1} / \Omega_{2}$, being $\Omega_{1}\left(\Omega_{2}\right)$ the tunneling rate between left and middle (middle and right) traps [2]. The SAP technique consists in lowering the barriers in a counterintuitive fashion (i.e., favoring first the tunneling between the middle and right traps and then the tunneling between the left and middle traps, such that $|D(\theta)\rangle$ transforms from $|L\rangle$ to $|R\rangle[2])$. Note that although the spatial dark state presents a node, the imperfect adiabatic evolution (due to being performed in a finite time) means that $\psi(x, t)$ during the evolution will not be exactly equal to the dark state, and thus present a quasinode (with a small population) instead of a node.

Thus, the temporal profile of the barriers height is taken to be [7]

$$
\begin{gathered}
V_{23}(t)= \begin{cases}\left(V_{\max }-V_{\min }\right)\left(\frac{2 t}{t_{\mathrm{p}}}-1\right)^{4}+V_{\min } & 0<t<t_{p} \\
V_{\max } & \text { elsewhere }\end{cases} \\
V_{12}(t)=V_{23}\left(t-t_{d}\right),
\end{gathered}
$$

being $t_{d}$ the time delay between the pulsing of $V_{23}$ and $V_{12}$, giving a total transport time $T=t_{p}+t_{d}$. For the numerical simulations, we take $V_{\min }=5 \hbar \omega_{x}, V_{\max }=10^{3} \hbar \omega_{x}$, $\sigma=0.16 \alpha^{-1}, x_{b}=0.48 \alpha^{-1}$, and $t_{d}=0.15 t_{p}$. We will study the effect of slowing down the process by varying the pulsing time $t_{p}$. For $\omega_{x} t_{p}<1000$ the transport breaks down since it is not adiabatic enough.

\section{NUMERICAL SIMULATIONS}

We show in Fig. 1(a) the probability distribution during the BEC transport for $g=0$ with the barrier heights from Eqs. (9) 


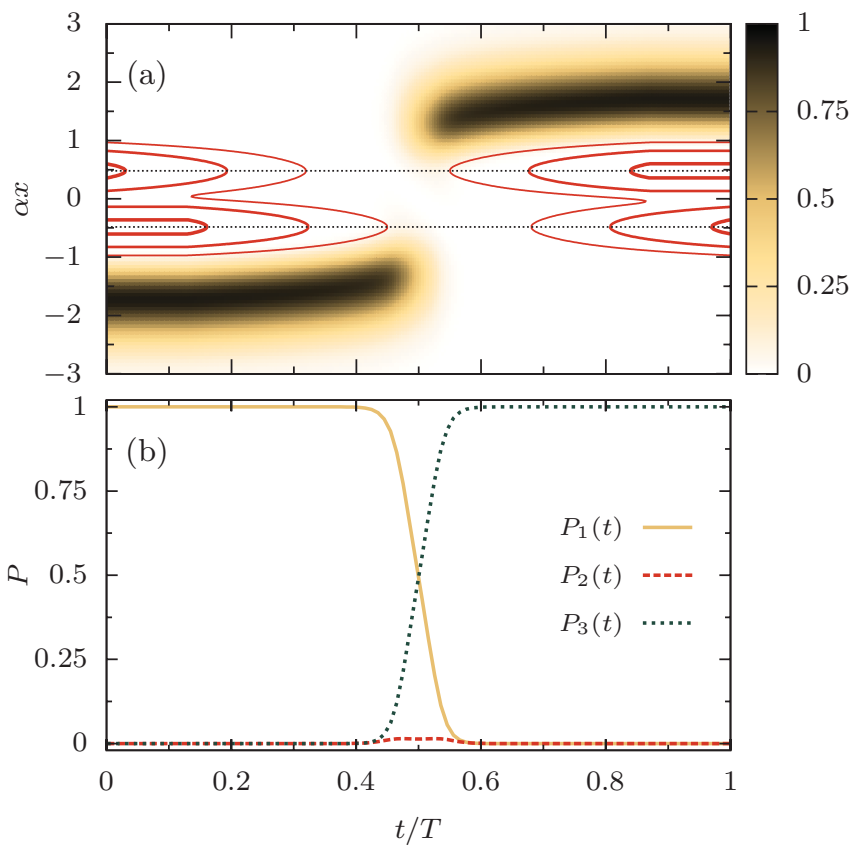

FIG. 1. (Color online) Adiabatic BEC transport for $g=0$ and $\omega_{x} t_{p}=5000$. (a) Atomic probability density $|\psi(x, t)|^{2}$, potential contour lines for $V(x, t)=10 \hbar \omega_{x}, 100 \hbar \omega_{x}$, and $750 \hbar \omega_{x}$ (solid lines, from thinner to thicker), and barrier positions $x= \pm x_{b}$ (dotted lines). (b) Region populations over time.

and (10). Indeed, when the potential barriers are lowered in the counterintuitive fashion of the SAP technique, the condensate vanishes from the left region and appears in the right region with a very small population in the space between the barriers. This is validated by Fig. 1(b) that shows that, while the BEC population is completely transferred to the right region, the middle region remains unpopulated the entire time due to the fact that the spatial dark state that the system is (ideally) following presents a node in this region.

To elucidate the transport process we have computed a set of Bohmian trajectories associated to this evolution, shown in Fig. 2(a). The trajectories distribution follows the wave function at all times, starting in the left trap and ending in the right one. All trajectories cross the middle region, but to keep a low population in the middle region they are forced to increase their velocity [5]. We compute the quasinode position (the dark state node position) $x_{n}(t)$ as the position in the central region where the population is minimum, and see that the trajectories accelerate when they get closer to it, reaching velocities much larger than the mean wave-packet velocity, see Fig. 2(b). From a Bohmian point of view, one can say that the trajectories in the quasinode borrow their energy from the quantum potential. Since the probability presence in the quasinode is very small, the trajectories there will have a small contribution to average values. Note that the mean kinetic energy remains very low since each trajectory velocity peaks at a different time $t_{n}\left(x_{0}\right)$, corresponding to the time at which the trajectory starting at $x_{0}$ crosses the quasinode [i.e., $\left.\mathrm{x}\left[t_{n}\left(x_{0}\right), x_{0}\right]=x_{n}\left(t_{n}\left(x_{0}\right)\right)\right]$. However, the process is not marginal since all trajectories exhibit the same dynamics and reach similar velocities, corresponding to $v\left(x_{n}(t), t\right)$, the dashed line in Fig. 2(b).

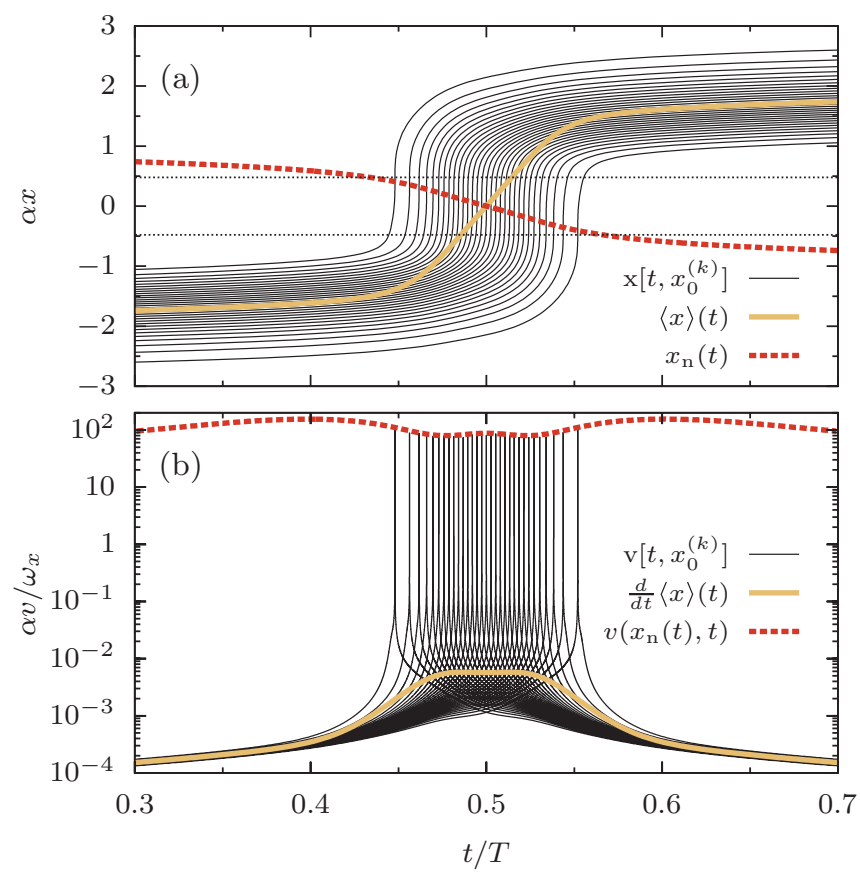

FIG. 2. (Color online) (a) Set of 30 Bohmian trajectories $\mathrm{x}\left[t, x_{0}^{(k)}\right]$ associated to the evolution shown in Fig. 1(a) (black lines), atomic mean position (solid line), quasinode position $x_{n}(t)$ (dashed line), and barrier positions $x= \pm x_{b}$ (dotted lines). (b) Bohmian velocities $\mathrm{v}\left[t, x_{0}^{(k)}\right]$ of the trajectories in (a) (black lines), atomic mean velocity (solid line), and Bohmian velocity at the quasinode position (dashed line). The distribution of initial positions $x_{0}^{(k)}$ follows $|\psi(x, 0)|^{2}$.

As we already mentioned, since the transport is performed in a finite time, the dynamic state $\psi(x, t)$ will not be exactly equal to the dark state, and therefore it will present a nonzero atomic population at the spatial dark state node position. If the evolution is made more adiabatic (i.e., more slowly), the dynamic state will remain closer to the dark state and the quasinode population will be smaller. This will result in an increase of the trajectories velocities at the quasinode [cf. Eq. (3)], presenting a surprising effect: by slowing down the total SAP sequence it is possible to achieve ultrahigh velocities.

We have computed the ensemble average of the velocity that the trajectories achieve at the quasinode $\left\langle v_{\max }\right\rangle$. By using Eqs. (3) through (5) and $\mathrm{v}\left[t, x_{0}\right] \geqslant 0$ and $\dot{x}_{n}(t) \leqslant 0$ [cf. Fig. 2(a)], such an average reads

$$
\begin{aligned}
\left\langle v_{\max }\right\rangle= & \int_{\mathcal{C}_{1}} d x_{0}\left|\psi\left(x_{0}, 0\right)\right|^{2} \mathrm{v}\left[t_{n}\left(x_{0}\right), x_{0}\right] \\
= & \int_{\mathcal{C}_{1}} d x_{0} \int_{0}^{T} d t\left|\psi\left(x_{0}, 0\right)\right|^{2} \mathrm{v}\left[t, x_{0}\right] \delta\left(t-t_{n}\left(x_{0}\right)\right) \\
= & \int_{0}^{T} d t \int_{\mathcal{C}_{1}} d x_{0}\left|\psi\left(x_{0}, 0\right)\right|^{2} \mathrm{v}\left[t, x_{0}\right] \\
& \times\left(\mathrm{v}\left[t, x_{0}\right]-\dot{x}_{n}(t)\right) \delta\left(x_{n}(t)-\mathrm{x}\left[t, x_{0}\right]\right) \\
= & \int_{0}^{T} d t\left[\frac{J\left(x_{n}(t), t\right)^{2}}{\left|\psi\left(x_{n}(t), t\right)\right|^{2}}-J\left(x_{n}(t), t\right) \dot{x}_{n}(t)\right]
\end{aligned}
$$

The second term in the last integral of Eq. (11) is orders of magnitude smaller than the first one since $\dot{x}_{n}$ is much smaller than the peak velocities [cf. Fig. 2(b)]. 

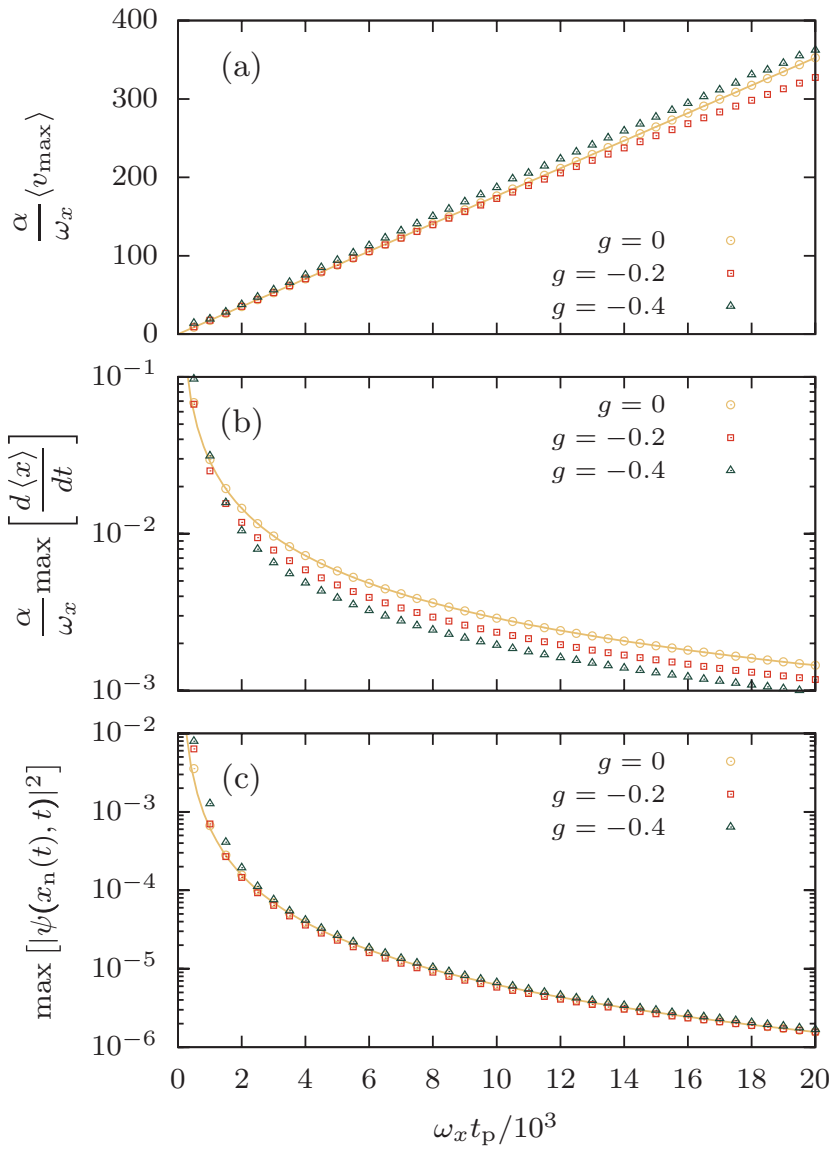

FIG. 3. (Color online) Results for different simulations with different values of $t_{p}$ and $g$. (a) Mean trajectory velocity at the quasinode. (b) Maximum mean atomic velocity. (c) Maximum probability density at the quasinode. Solid lines are, respectively, a linear fit, a $t_{p}^{-1}$ fit and a $t_{p}^{-2}$ fit for the $g=0$ data.

Figure 3 shows the result of various simulations for different times $t_{p}$ and different interaction strengths $g$. Variations in $g$ slightly change the values of the calculated quantities, but the overall behavior remains the same. Thus, for clarity, we have performed fits for the $g=0$ data only. Figure 3(a) shows that the mean velocity that the trajectories reach when passing through the quasinode grows linearly with $t_{p}$. Note that all trajectories accelerate abruptly, but at different times, and then the maximum value that the mean atomic velocity $\max [d\langle x\rangle / d t]$ takes during the evolution decreases inversely proportional to $t_{p}$ [see Fig. 3(b)] remaining orders of magnitude below $\left\langle v_{\max }\right\rangle$. There is no apparent limit to the Bohmian velocities in the middle region as the adiabaticity is increased and there will be a regime for (finite) $t_{p}$ where $\left\langle v_{\max }\right\rangle$ will approach and eventually surpass the speed of light. With the scalings shown in Fig. 3, an experiment performed in 60 seconds using a trap with $\omega_{x}=2 \pi \times 1$ $\mathrm{MHz}$ would result in a mean wave-packet velocity of around $19 \mathrm{~nm} / \mathrm{s}$, and a mean trajectory velocity at the quasinode of $1.6 \times 10^{6} \mathrm{~m} / \mathrm{s}$, which is almost at the relativistic regime. Note that while the Bohmian trajectories velocity is much smaller than the speed of light, the usual treatment with the TDSE is completely valid. Nevertheless, when such velocities are near or above the speed of light, relativistic corrections to the
TDSE are needed to properly address the transport and avoid superluminal propagation [10]. It is surprising that the TDSE (or the GPE) ceases to be valid in a system where the associated average velocities are small (and smaller as the adiabaticity is increased), and at variance with the usual tunneling problems, such high velocities here appear in a minimum of potential.

Figure 3(c) shows that the maximum population of the quasinode during the evolution decreases inversely proportional to $t_{p}^{2}$. This nonzero population appears due to an imperfect following of the dark state and its decrease is what makes the high velocities in the middle region to grow. Note that a perfect node would forbid the transport, and therefore a nonzero population is needed for the transport to take place. As expected, the computed flux through the quasinode $\int_{0}^{T} d t J\left(x_{n}(t), t\right)$ is 1 in all cases, meaning that the entire transported wave function is transiting through the quasinode.

\section{CONCLUSION}

In summary, we have studied the adiabatic transport of a single atom and a BEC in a triple well, where the system state is transferred from the left to the right well by following a spatial dark state that presents a node in the central region. Due to the imperfect following of the dark state, the node of the dynamical state will present some population that can be made arbitrarily small as the process is performed more slowly. By applying the (nonrelativistic) Bohmian formalism to this particular system we have shown that the vanishing atomic population in the vicinity of the quasinode leads to an increase of the trajectories velocities. Furthermore, as the process is performed more adiabatically, these velocities increase with no apparent limit, and in the regime where they approach (and surpass) the speed of light relativistic corrections should be taken into account to describe correctly the system dynamics. Without such relativistic corrections one could erroneously conclude that superluminal transport is possible. The simulation of (correct) relativistic Bohmian trajectories would be very computationally demanding, especially in the regime where (incorrect) superluminal Bohmian trajectories from the TDSE are expected to occur.

Finally, it would be interesting to investigate if the Doppler shift in the light absorption due to the atomic velocities could be used to (weakly) measure the atomic momenta and study some of the features described in this paper in similar lines as in Ref. [13], or whether the very high accelerations (and decelerations) during the SAP technique could lead to the emission of radiation when applied to charged particles.

\section{ACKNOWLEDGMENTS}

The authors gratefully acknowledge discussions with Albert Bramon, Alfonso Alarcón, Daniel Viscor, Gerhard Birkl, Luis Plaja, Luis Roso, and Maciej Lewenstein, and financial support through Spanish MICINN Contracts No. TEC2009-06986, No. FIS200802425, No. FIS2011-23719, and No. CSD2006-00019, and the Catalan Government Contracts No. SGR200900347 and No. SGR2009-00783. A. B. acknowledges financial support through Grant No. AP 2008-01275 from the MICINN. 
[1] K. Bergmann, H. Theuer, and B. W. Shore, Rev. Mod. Phys. 70, 1003 (1998).

[2] K. Eckert, M. Lewenstein, R. Corbalán, G. Birkl, W. Ertmer, and J. Mompart, Phys. Rev. A 70, 023606 (2004); K. Eckert, J. Mompart, R. Corbalán, M. Lewenstein, and G. Birkl, Opt. Commun. 264, 264 (2006).

[3] T. Opatrný and K. K. Das, Phys. Rev. A 79, 012113 (2009).

[4] S. McEndoo, S. Croke, J. Brophy, and T. Busch, Phys. Rev. A 81, 043640 (2010).

[5] A. Benseny, S. Fernández-Vidal, J. Bagudà, R. Corbalán, A. Picón, L. Roso, G. Birkl, and J. Mompart, Phys. Rev. A 82, 013604 (2010).

[6] E. M. Graefe, H. J. Korsch, and D. Witthaut, Phys. Rev. A 73, 013617 (2006); M. Gajdacz, T. Opatrný, and K. K. Das, ibid. 83, 033623 (2011).
[7] M. Rab, J. H. Cole, N. G. Parker, A. D. Greentree, L. C. L. Hollenberg, and A. M. Martin, Phys. Rev. A 77, 061602R (2008).

[8] D. Bohm, Phys. Rev. 85, 166 (1952); Applied Bohmian Mechanics: From Nanoscale Systems to Cosmology, edited by X. Oriols and J. Mompart (Pan Standford Publishing, Singapore, 2012).

[9] P. R. Holland, The Quantum Theory of Motion: An Account of the De Broglie-Bohm Causal Interpretation of Quantum Mechanics (Cambridge University Press, Cambridge, England, 1995).

[10] C. R. Leavens and R. Sala Mayato, Ann. Phys. (Leipzig) 7, 662 (1998).

[11] W. Struyve, W. De Baere, J. De Neve, and S. De Weirdt, Phys. Lett. A 322, 84 (2004).

[12] L. Pitaevskii and S. Stringari, Bose-Einstein Condensation (The International Series of Monographs on Physics) (Oxford University Press, New York, 2003).

[13] S. Kocsis et al., Science 332, 1170 (2011). 